\title{
Kernos
}

Revue internationale et pluridisciplinaire de religion grecque antique

8 | 1995

Varia

\section{La traduction de textes religieux dans l'Égypte gréco-romaine}

\section{Bruno Rochette}

\section{(2) OpenEdition \\ Journals}

Édition électronique

URL : http://journals.openedition.org/kernos/599

DOI : $10.4000 /$ kernos.599

ISSN : 2034-7871

Éditeur

Centre international d'étude de la religion grecque antique

Édition imprimée

Date de publication : 1 janvier 1995

Pagination : 151-166

ISSN : 0776-3824

Référence électronique

Bruno Rochette, "La traduction de textes religieux dans l'Égypte gréco-romaine », Kernos [En ligne], 8 | 1995, mis en ligne le 11 avril 2011, consulté le 19 avril 2019. URL : http://journals.openedition.org/ kernos/599; DOI : 10.4000/kernos.599 


\title{
La traduction de textes religieux dans l'Ëgypte grẻco-romaine
}

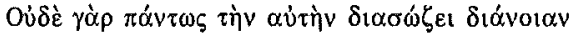

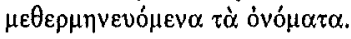

JAMBLIQUE, Mystères d'Égypte, VII, 5

Parmi les nombreux problèmes que pose la traduction dans l'antiquité gréco-romaine ${ }^{1}$, il en est un qui soulève des difficultés particulièrement aiguës : la traduction de textes sacrés. En effet, traduire un texte à contenu religieux, ce n'est pas traduire un document officiel ou juridique ${ }^{2}$ ou même rendre dans une autre langue une œuvre littéraire profane ${ }^{3}$. Dans un texte sacré, le mot recèle une force qui agit sur la divinité, «la parole (...) devient une puissance, une force, une action $»^{4}$. Le traduire, c'est livrer à l'étranger, - au $\beta \alpha \dot{\alpha} \rho \beta \alpha \rho \circ \zeta$, selon la conception grecque, - qui est aussi quelquefois l'ennemi, l'efficacité de la parole rituelle, car « la parole est moins considérée en elle-même que replacée dans l'ensemble d'une conduite dont les valeurs symboliques convergent $»^{5}$. Traduire un texte sacré, dans lequel une sorte de lien mystique unit le signifiant et le signifié, c'est lui faire perdre sa force religieuse et l'effet de la parole-acte, exprimée dans un langage destiné aux seuls dieux ${ }^{6}$. La première partie du

1 Nous renvoyons à notre étude Du grec au latin et du latin au grec. Les problèmes de la traduction dans l'antiquité gréco-romaine, à paraître dans Latomus. En outre, pour un aperçu des problèmes que pose la traduction des textes liturgiques, on verra M. TESTARD, $\grave{A}$ propos de la traduction française des textes liturgiques, in BAGB (1966), p. 506-509 et A. RONCONI, Del tradurre $i$ testl liturgtel, in Interpretaztont grammaticali, Roma, 197122, p. 137-166.

2 Sur cet aspect, voir W. PEREMANS, Les épunvếs dans l'Égypte gréco-romalne, in Das römiscb-byzantinische Ägpten. Akten des int. Symposiums 26. - 30. September 1978 in Trier, Mainz, 1983 (Ägyptiaca Treverensia, 2), p. 11-17 et nos études intitulées Traducteurs et traductions dans l'Égypte gréco-romaine, à paraître dans la Chronique d'Égypte et Sur le bilingutsme dans l'Égypte gréco-romaine, à paraître dans cette même revue.

3 Voir notre étude Les traductions grecques de l'Énéide sur papyrus. Une contribution à l'étude du bilinguisme gréco-latin au Bas-Empire, in $L E C, 58$ (1990), p. 333-346.

4 M. DETIENNE, Les maîtres de vérttê dans la Grèce arcbatque, Paris, 1967, p. 53-54. Le

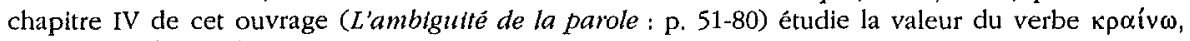
qui désigne la parole-acte.

5 M. DETIENNE, op, cit. (n. 4), p. 51. 
dialogue entre Socrate et Hermogène dans le Cratyle de Platon développe ce thème $e^{7}$. Socrate donne raison à Cratyle lorsqu'il affirme « qu'il n'est pas donné à tout le monde d'être un artisan de noms, mais à celui-là seulement qui, les yeux fixés sur les noms naturels de chaque objet, est capable d'en imposer la forme aux lettres et aux syllabes $»^{8}$.

L'Égypte gréco-romaine, qui constitue un véritable point de rencontre entre les cultures, - au moins trois civilisations s'y côtoyaient dès l'époque hellénistique, l'égyptienne, la juive et la grecque, - sera confrontée à maintes reprises au dilemme «traduire ou ne pas traduire ${ }^{9}$. Dans les milieux sacerdotaux d'Égypte, on constate deux attitudes opposées face à la traduction des textes théologiques, dont nous allons tenter d'étudier ici les manifestations littéraires et le rôle dans la diffusion de la théologie égyptienne. L'une est hostile aux entreprises de traduction. Elle refuse de traduire, car faire passer un texte religieux dans une autre langue, c'est faire perdre aux mots leur force et faire disparaître l'auréole que confère à la civilisation égyptienne l'ancienneté. L'autre, en revanche, accepte de traduire. Procédant d'une volonté missionnaire et apostolique, ce courant favorable à la traduction, - non sans certaines réserves toutefois, ainsi que nous le verrons, - a pour but ultime de communi-

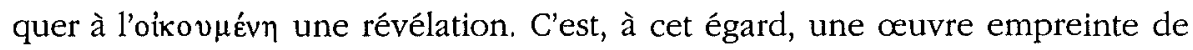
grande générosité. L'adaptation de la théologie égyptienne à la pensée grecque, presque totalement fermée aux langues étrangères ${ }^{10}$, suppose en effet une traduction de l'égyptien en grec. Nous ignorons toutefois les modalités de cette opération, mais nous sommes à même d'en apprécier le résultat et d'en percevoir les restrictions.

6 W. GÜNTERT, Von der Sprache der Götter und Geister, Halle, 1921 et W. THEILER, Die Spracbe des Getstes in der Antike, in Spracbgescbicbte und Wortbedeutung. Festscbrift $A$. Debrunner, Bern, 1954, p. 431-440 [= Forschungen zum Neuplatonismus, Berlin, 1966, p. 302-312].

7 385a1-390e4. Cf. P.M. GENTINETTA, Zur Spracbbetracbtung bet den Sopbisten und in der stolsch-bellentstischen Zeit, Diss. Zürich, Winterthur, 1961, p. 47-49.

8 390e1-4. Nous citons la traduction de L. Méridier (Paris, 1931).

9 Cl. PRÉAUX, De la Grèce classique à l'époque bellénistique. Tradulre ou ne pas traduire, in $C E, 42$ (1967), p. 369-382 [article dont on trouvera la substance dans Le monde belléntstique. La Grèce et l'Orlent (323-146 av. J.-C.), II, Paris, 1978 (Nouvelle Clio, 6bis), p. 552-553); D.N. WIGTIL, The Translation of Religious Texts in the Graeco-Roman World, Diss. Univ. of Minnesota, Minneap. 1980 [non vidimus] (résumé in DA 41 [1981], 4703 A) et J. SCHWARTZ, Traductions en Égypte gréco-romaine, in Mélanges $P$. Lévêque, Il (antbropologie et société), Paris, 1989, p. 380386.

10 J. WERNER, Nichtgriechische Sprachen im Bewusstsein der antiken Griecben, in Festschrift R. Muth, Innsbrück, 1983, p. 584-595. - Selon la tradition (DIOGÈNE LÄ̈RCE, VIII, 3), Pythagore aurait connu l'égyptien. C'est là une fiction pure et simple. 


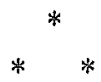

Le courant défavorable à l'acte de traduire nous met d'emblée en contact avec un des grands monuments de la traduction d'une langue étrangère en grec, réalisé en terre égyptienne : la version grecque de la Bible hébraïque connue sous le nom de «traduction des Septante». Le pseudépigraphe, en réalité anonyme, qu'est la Lettre d'Aristée à Philocrate (IIIe/Ile siècle av. J.-C.) donne à penser que cette versio Graeca des livres sacrés, qui devait rester unique, ne fit pas l'unanimité parmi les Juifs des communautés égyptiennes ${ }^{11}$. Elle dut certainement susciter chez les traducteurs un malaise comparable à celui que connaîtra saint Jérôme, bien des siècles plus tard, lorsqu'il entreprit la traduction en latin du Texte Sacré, ubi et uerborum ordo mysterium est, selon l'expression qu'il emploie lui-même dans la Lettre à Pammachius ${ }^{12}$. La légende de la Septante telle que la rapporte la Lettre d'Aristée à Pbilocrate est bien connue $^{13}$. À l'initiative de Ptolémée II (285-247), - ou de son bibliothécaire, Démétrios de Phalère, - qui voulait disposer d'une traduction des cinq premiers livres de la Bible hébraïque pour la bibliothèque du Musée, on réunit 72 traducteurs (six par tribu d'Israël) qui travaillèrent séparément dans l'île de Pharos, en face d'Alexandrie. Une fois leur travail terminé, ils confrontèrent leurs traductions et s'avisèrent qu'elles étaient identiques, preuve que leur travail était inspiré. En réalité, cette traduction fut une œuvre de longue haleine, dont les débuts ne sont pas datables, mais dont on peut situer l'achèvement vers 132 . Elle fut aussi le fruit d'une collaboration importante, et on peut imaginer sans peine que certains livres connurent plusieurs versions. Quant à la prétendue volonté d'enrichir la bibliothèque du Musée par Ptolémée II, qui aurait été le commanditaire de la traduction, elle relève aussi de la légende. En fait c'est un besoin tout pratique qui fut à l'origine de cette traduction. Les Juifs des communautés d'Égypte en étaient venus à ignorer l'hébreu et à ne plus parler que la ko vín hellénistique ${ }^{14}$. Ces Juifs avaient besoin d'un texte compréhensible des Écritures, spécialement du Pentatenque, pour la célébration des offices. C'est donc indirectement et aussi, d'une certaine façon, involontairement, que la

11 314-316. Voir l'édition d'A. Pelletier (Paris, 1962 = Sources chrétiennes, 89).

12 Lettres, LVII (de optimo genere interpretand), 5 (sur saint Jérôme traducteur, voir, dans une bibliographie abondante, F. BLATT, Remarques sur l'histoire des traductions latines, in CEM, 1 [1938], p. 220).

$13 \$ 10-11$.

14 J.A. SOGGIN, Billinguismo o trilinguismo nell'ebraismo post-esilico. Il caso dell'aramaico $e$ del greco, in Vicino Oriente 3 (1980) [Atti del seminario di studt su bilinguismo e traduzioni nell'antico Orlente], p. 199-207 [= Il bilinguismo degli Anticht, Genova, 1991, p. 83-94]. 
Bible passa dans la pensée grecque. D'autre part, ce passage des Écritures Saintes par l'Égypte laissa, selon les spécialistes, des traces qui sont perceptibles dans le texte grec. S. Morenz relève dans la version grecque, notamment dans le récit de la Création (Maasé Bereschit), des notions cosmologiques propres au clergé hermopolitain ${ }^{15}$, et M. Görg, de son côté, croit percevoir dans la traduction une influence de la pensée platonicienne ${ }^{16}$.

Dans la littérature égyptienne, un texte témoigne des hésitations du traducteur devant un livre sacré remontant à Nectanébo ${ }^{17}$. Il s'agit d'une arétalogie du dieu guérisseur Imouthès, assimilé à Asclépios, connue par un papyrus d'époque romaine (IIe siècle ap. J.-C.). Après avoir hésité plusieurs fois et s'être remis à la besogne, ému par la grandeur et la majesté du récit, le traducteur se sent finalement moralement tenu, après la guérison de sa mère et de lui-même, d'achever le travail entrepris et de faire connaître ainsi aux Grecs la révélation divine que contient ce livre sacré. Examinons les deux passages du texte où se font jour les hésitations du traducteur.

Col. II, 1. 32-42 :

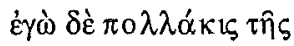

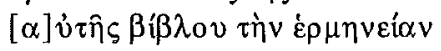

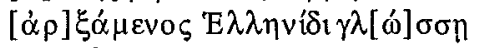

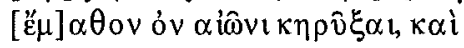

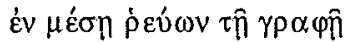

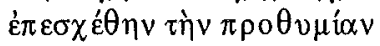

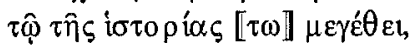

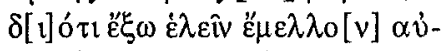

$\tau \eta^{\prime} v \cdot \theta \varepsilon[0 \hat{\imath}] \varsigma \gamma \dot{\alpha} \rho \mu o ́ v o r[s] \dot{\alpha} \lambda \lambda \lambda^{\prime}$ ov่

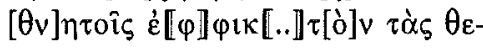

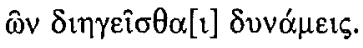

Moi, après avoir plusieurs fois commencé la traduction du même livre en grec, j'appris à la longue comment le proclamer, mais, tandis que j'étais en plein travail d'écriture, je fus arrêté dans mon ardeur par la grandeur du récit, car j'étais sur le point de le rendre public. C'est que seuls les dieux, et non les mortels, ont le droit de raconter les hauts faits des dieux.

15 Ägyptiscbe Spuren in der Septuaginta, in Mullus. Festschrfft Th. Klauser, Münster, 1964, p. 250-258 [= Religion und Gescbicble des alten Ägyten, Köln-Wien, 1975, p. 417-428].

16 Ptolemaiscbe Theologie in der Septuaginta, in H. Maehler et V.M. Strocka (éds), Das ptolemalsche Ägypten. Akten des int. Symposiums 1976 zu Berlin, Berlin, 1978, p. 178 et 181 [= Kairos, 20 (1978), p. 208-217].

$17 P$. Oxy. 11.1381, spéc. 1. 32-42 et 191-202 [= G. MANTEUFFEL, De opusculis Graecis Aegyptle papyris, ostracis lapidibusque collectis, II, Warszawa, 1930, p. 86-91]. 
Col, LX, 1. 191-202:

$[\pi \hat{\alpha}] \sigma \alpha \gamma \dot{\alpha} \rho[\dot{\alpha}] v \alpha-$

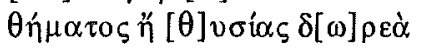

$\tau o ̀ v \pi \alpha \rho \alpha v \tau[\hat{i}] \kappa \alpha \mu[o ́] v[0] v$

$\dot{\alpha} \kappa \mu \alpha \dot{\zeta} \zeta \varepsilon \iota \alpha[\uparrow]$ [

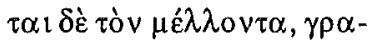

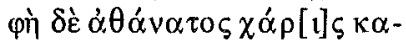

$\tau \dot{\alpha} \kappa \alpha$ pòv $\dot{\alpha} v \eta \beta \dot{\alpha} \sigma \kappa[\mathrm{o}] v \sigma \alpha$

$\tau \grave{\eta}[v] \mu v \eta \dot{\eta} \mu \nu$. 'Е $\lambda \lambda \eta \nu[\hat{]}] \varsigma \delta \dot{\varepsilon}$

$\pi[\hat{\alpha}] \sigma \alpha \gamma \lambda \hat{\omega} \sigma \sigma \alpha$ iทे $\sigma \dot{\eta} v \lambda \alpha-$

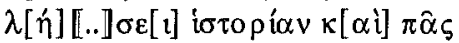

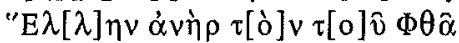

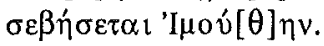

Car chaque présent que constitue une offrande ou un sacrifice compte seulement pour le moment présent et n'est rien pour le futur, mais un écrit est un mémorial immortel de gratitude qui ravive, quand il faut, le souvenir. Toute langue grecque racontera ton histoire et tout Grec honorera le fils de Ptah, Imouthès.

Le même sentiment et le même scrupule à traduire se retrouvent dans les traités réunis sous le nom d'Hermès Trismégiste ${ }^{18}$. Le traité XVI contient un discours adressé par l'Asclépios d'Égypte au roi Ammon prescrivant qu'il n'y ait jamais de traduction en grec $^{19}$. L'argument avancé par l'auteur est que la pensée deviendrait obscure lorsque, plus tard, les Grecs voudront la traduire dans leur langue. Jugeons-en par le texte.

Pour autant donc que tu en aies le pouvoir, ô roi, - et tu peux tout, - préserve bien ce discours de toute traduction, afin que de si grands mystères ne parviennent point jusqu'aux Grecs et que l'orgueilleuse élocution des Grecs, avec son manque de nerf et ce qu'on pourrait dire ses fausses grâces, ne fasse point pâlir et disparaître la gravité, la solidité, la force active des vocables de notre langue. Car les Grecs, ô roi, n'ont que des discours vides, bons à produire des démonstrations : et c'est là en effet toute la philosophie des Grecs, un bruit de mots. Quant à nous, nous n'usons pas de simples mots, mais de sons tout remplis d'efficace.

(Traduction A.-J, Festugière)

18 XVI, 2 ; XVIII, 16.

19 A.D. NOCK et A.J. FESTUGIÈre, Hermès Trismégiste, Corpus Hermeticum, II (Paris, 1960), Traité XVI, 2. L'inspiration égyptienne du Corpus Hermeticum a été étudiée par Ph. DERCHAIN, L'autbenticité de l'inspiration égyptienne dans le Corpus Hermeticum, in RHR, 161 (1962), p. 175198. 
Peut-être peut-on rapprocher, avec J. Schwartz, l'affabulation du traité XVI, qui évoque un roi, des sentiments hostiles des Juifs d'Égypte envers Ptolémée II et la Septante.

Cette hostilité à la traduction aura longue vie. Elle conduira à la constitution d'une religion ésotérique, qui ne sortira pas du cercle des initiés, et débouchera sur la gnose et ses secrets, dont les doctrines, fortement teintées d'emprunts aux religions orientales, ne survivront cependant pas au triomphe du christianisme ${ }^{20}$. Elle inspirera encore au début du IVe siècle le traité de Jamblique intitulé les Mystères d'Égypte ${ }^{21}$, qui souligne l'inefficacité des paroles rituelles une fois traduites et qui recommande les prières en langue originale.

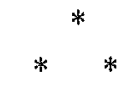

Ce courant conservateur connaîtra toutefois sa contrepartie. Procédant d'une intention apostolique, le courant favorable à la traduction contribue au passage de la religion égyptienne dans la pensée grecque. Les textes traduits sont en général des petits récits qui illustrent des visions, qu'elles soient accompagnées de guérissons, qu'elles aient trait à des actes du culte ou qu'elles mettent en scène une divinité qui communique un art ou une science ${ }^{22}$.

L'exemple le plus éclairant est le récit connu sous le nom de Songe de Nectanébo (IIe siècle av. J.-C.) découvert sur un papyrus provenant du Serapeum de Memphis ${ }^{23}$. Le récit décrit Isis sur son trône, entourée des dieux égyptiens, et Onouris venant se plaindre de l'inachèvement du temple dédié à Nectanébo. - On citera aussi une inscription du Ile siècle ap. J.-C., connue sous le nom de Vision du dieu Mandoulis, gravée par un pèlerin sur un mur du temple de Kalabcha, l'antique Talmis, en Nubie, qui donne l'ordre au dieu

20 A.-J. FESTUGIÈRE, L'bermétisme, in Hermétisme et mystique patenne, Paris, 1967, p. 28-87 et J.E. MÉNARD, Mythes, mystères et gnose, in Ktèma, 7 (1982), p. 75-85. - La bibliographie est réunie par A. GONZÁLEZ BLANCO, Hermetism. A Brbliographical Approach, in $A N R W$, II, 17/4, Berlin-New York, 1984, p. 2240-2281.

21 VI, 7 («les Chaldéens ont isolé, pour qu'il reste pur, le langage destiné aux seuls dieux »); VI, 5 (« d'où il appert combien on a raison de préférer la langue des peuples sacrés à celles des autres hommes ; car, à être traduits, les noms ne conservent pas entièrement le même sens : chaque peuple a des caractéristiques impossibles à transposer dans la langue d'un autre; ensuite, même si on peut traduire ces noms, en tout cas ils ne gardent pas la même puissance ; de plus, les noms barbares ont à la fois beaucoup de solennité et beaucoup de concision (...) »). (Traduction E, des Places [Paris, 1966]).

22 A.-J. FESTUGIÈRE, La révélation d'Thermès Trismégiste, I (L'astrologie et les sciences occultes), Paris, 1950, p. 52-66.

$23 U P Z$, I, 81 (avec la bibliographie et un commentaire important) [= G. MANTEUFFEL, De opusculis Graecis, II, p. 112-116] et A.-J. FESTUGIÈRE, Hermès Trismégiste, I, p. 55-56. 
Mandoulis de chanter ses vertus en langue grecque plutôt que dans «le langage barbare des Éthiopiens " ${ }^{24}$. Ce dieu indigène, adoré dans cet endroit désert, appelé par les Grecs Mandoulis, était assimilé à Horus, fils d'Isis, ou au Soleil, ou même au fils de Latone, Apollon. Commencé sous Auguste, le nouveau temple ne fut terminé qu'à la fin du Ier siècle, sous Vespasien. Les pèlerins, qui venaient en grand nombre dans cet endroit retiré, où il n'y avait guère qu'une garnison romaine qui veillait sur la frontière, avaient l'habitude de graver ou de peindre sur les murs extérieurs du temple des formules d'adoration où ils demandaient au dieu Mandoulis de les protéger, eux-mêmes et leur famille.

Le genre littéraire de l'arétalogie, qui fleurit à travers tout le monde grec $^{25}$, favorisera aussi les entreprises de traduction, ce qui constituera un excellent canal de diffusion parmi les Grecs des révélations de la théologie égyptienne. C'est bien sûr Isis, promise par les Égyptiens à la puissance universelle, qui est la divinité le plus célébrée de cette manière ${ }^{26}$. Rien d'étonnant que le récit de ses hauts faits passe, de la volonté même des Égyptiens, dans les autres cultures. On trouve la litanie de ses vertus en grec inscrite au temple de Medinet-Madi dès le premier siècle ap. J.-C. ${ }^{27}$, ainsi que sur un papyrus d'Oxyrynchus du Ile siècle ap. J.-C. ${ }^{28}$

Enfin, une dernière catégorie de textes constitua un matériau particulièrement adapté à la traduction ou plutôt, dans ce cas précis, à la rencontre des trois cultures présentes en terre égyptienne. Ils s'agit des papyrus magiques, qui sont le reflet de la religiosité des couches inférieures de la population. Certains d'entre eux se distinguent des écrits magiques en démotique. Ils comportent des éléments provenant de la Genèse en grec, - d'après la version des Septante ou une autre, - des translittérations de bribes de prières en

24 A.D. NOCK, $A$ vision of Mandulis Aion, in HThR, 27 (1934), p. 53-104 et A.-J. FESTUGİ̀RE, Hermès Trismégiste, I, p. 46-47. Voir encore A.-J. FESTUGIÈRE, L'expérience religieuse du médecin Thessalos, in $R B i, 48$ (1939), p. 45-77 [il s'agit d'une lettre adressée par un médecin philosophe, probablement Thessalos de Tralles, à Claude ou à Néron, qui contient le récit d'une vision au cours de laquelle l'auteur a obtenu de voir un dieu grâce aux pratiques magiques d'un prêtreprestidigitateur].

25 M. MALAISE, Les conditions de pénétration et de diffusion des cultes égyptiens en Italie, Leiden, 1972 (EPRO, 22), p. 173-174; J. LECLANT, Aegyptiaca et milieux isiaques. Recherches sur la diffusion du matériel et des idées isiaques, in ANRW, II, 17/3, Berlin-New York, 1984, p. 1692-1709 et L.V. ZABKAR, Hymns to Ists in ber Temple of Pbilae, Hanover-London, 1989.

26 A.J. FESTUGIÈRE, À propos des arétalogies d'Isis, in $H T h R, 42$ (1949), p. 209-234 [=Études de religion grecque et bellénistique, Paris, 1972, p. 138-163] et A. MÜLLER, Agypten und die griechiscbe Isis-Aretalogie, Berlin, 1961 ( $A S A W, 53 / 1)$.

$27 S E G$, VIII, 548-551 et XV, 860 (compléments bibliographiques et corrections).

28 P.Oxy. 11.1380 [= G. MANTEUFFEL, De opusculis Graecis, II, p. 70-85]. 
hébreu et même la transcription en caractères grecs de termes égyptiens. Ces textes bigarrés unissent ainsi trois civilisations et trahissent l'existence, dans les couches les plus basses de la population d'Égypte, d'un trilinguisme : grecégyptien-hébreu. Cette rencontre de trois pensées religieuses différentes, qui s'accompagne d'un trilinguisme, débouchera sur un véritable syncrétisme, caractéristique de la religion populaire, comme le montre le texte papyrologique. Il se présente comme un hymne litanique au dieu Créateur, dont les invocations ne sont pas sans rappeler celles que l'on trouve dans l'Hymne à Zeus de Cléanthe ou dans les hymnes philosophiques de Proclos.

PGM V 460-487 Preisendanz :

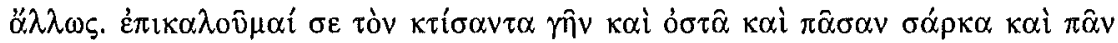

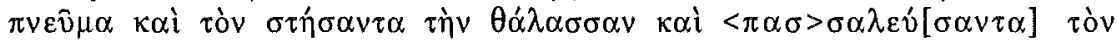

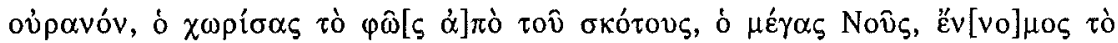

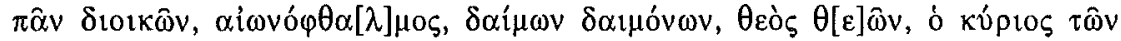

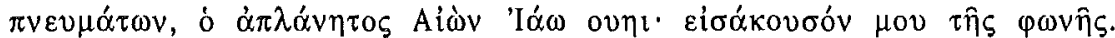

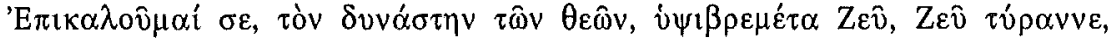

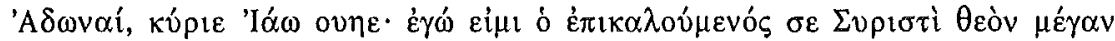

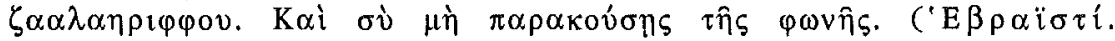

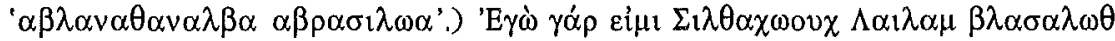

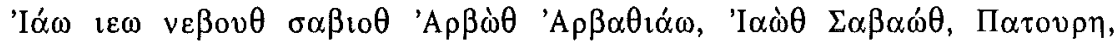

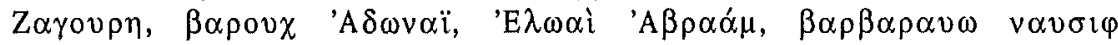

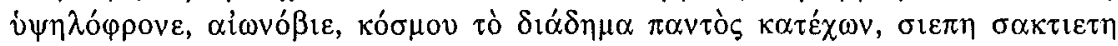

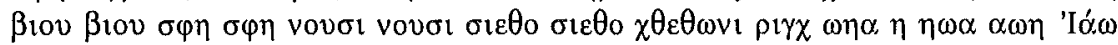

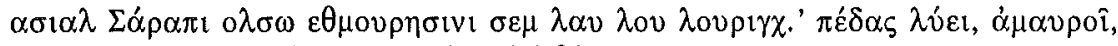

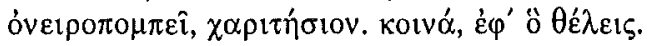

Une autre (prière). «Je t'invoque, toi qui as fait la terre, les os, toute chair et tout esprit, toi qui as créé la mer et qui as fixé le ciel, toi qui as séparé la lumière de l'obscurité, le Grand Esprit, toi qui, gardien de la loi, diriges le tout, oeil immortel, Démon des démons, Dieu des dieux, le Maître des esprits, l'Éternel qui ne dévie pas, Iaô oyêi, écoute ma voix. Je t'invoque, le Maître des dieux, Zeus qui gronde au haut du ciel, Zeus Roi, Adônai, Seigneur Iaô oyêt. Je suis celui qui t'invoque, en syriaque, comme Dieu grand. Ne dédaigne pas ma voix (en hébreu : ablanathanalba Abrasilôa). Car je suis : Silthachôouch, Lailam, Blasalôth, Iaô, Ieô, Nebouth, Sabioth, Arboth, Arbathiaô, Iaôth, Sabaôth, Patourê, Zagourê, Barouch, Adônai, Elôai, Abrabam, Barbarauô, Nausiph, toi aux pensées élevées, l'Éternel, toi qui tiens le diadème du monde entier, Siepê, Saktietê, Biou, Biou, Spbê, Spbê, Nousi, Nousi, Stetbo, Sietbo, Chtbethôni, Rigch, ôêa ê aôe aôê Iaô, Asial, Sarapi, Olsô, Etbmourêsimi, Sem, Lau, Lou, Lourigch.' Brise mes liens, rends-moi invisible, envoie-moi des songes. Supplication. Selon ton bon plaisir, comme tu le veux. 
En conclusion de l'étude qu'il consacre à ce texte curieux ${ }^{29}, M$. Philonenko tente de cemer la personnalité de l'auteur. S'il a encore quelque connaissance de l'hébreu et s'il parle aussi l'égyptien, c'est la Bible grecque qu'il connaît et lit. Son origine juive ne semble faire aucun doute, mais il est hellénisé et égyptianisé. Peut-être appartient-il, suppose $M$. Philonenko, à une des communautés juives d'Égypte encore vivantes après le règne d'Hadrien.

Le dossier des témoignages ainsi constitué, il s'agit de déterminer maintenant quels milieux représentent ces deux attitudes opposées. Ce problème trouve sa solution dans la nature même des textes traduits et dans le type de pensée qui anime les traducteurs. En effet, ces deux courants antithétiques procèdent de deux schémas de pensée complètement différents. L'un, qui refuse de traduire, attribue à la parole une valeur en soi et considère que la traduire, c'est la dénaturer et lui faire perdre son efficacité. C'est cette conception qui empêche la traduction des formules d'imprécation ou de serment. L'autre, favorable à la traduction, conçoit une vérité universelle qui transcende les langues et qui conserve sa valeur quel que soit l'idiome dans lequel elle est exprimée.

Le courant défavorable à la traduction est une manifestation de la croyance, déjà attestée dans la Grèce archaïque ${ }^{30}$, que le mot, considéré comme une succession de signes que sont les lettres, a une valeur intrinsèque qui lui confère une force opérante. L'étrangeté et le mystère qui entourent les $\xi_{\text {Evik }}$ óvó $\mu \alpha \tau \alpha^{31}$ confèrent aux idiomes barbares une efficacité que neutralise la traduction. Ainsi, selon la conception égyptienne, dans les prières magiques, la divinité est obligée d'obéir si on l'interpelle sous son nom indigène. Comme l'écrit F. Cumont ${ }^{32}$, «Il [le rite chez les Égyptiens] a une force opérante par lui-

29 M. PHILONENKO, Une prière magique au dieu Créateur (PGM 5, 459-489), in CRAI (1985), p. 433-452 et H.D. BETZ, The Greek Magical Papyri in Translation, Chicago-London, 1986, p. 109110. On peut rapprocher ce texte d'un passage de Lucien (Pbilospeudès, 31), où le pythagoricien Arignote fait une lecture incantatoire de textes magiques démotiques.

30 M. DETIENNE, op. cit. (n. 4), p. 80 : «pour que la philosophie pose le problème des rapports entre la parole et la réalité, pour que la Sophistique et la Rhétorique construisent une théorie du langage comme instrument de persuasion, il faut que soit consommée la ruine d'un système de pensée où la parole est prise dans un réseau de valeurs symboliques, où la parole est naturellement une puissance, une réalité dynamique, où cette parole, en tant que puissance, agit spontanément sur autrui ».

31 P. M. GENTINETTA, op. cit. (n. 7), p. 54-56.

32 F. CUMONT, Les religions orlentales dans le paganisme romain, Paris, $1929^{4}$, p. 87. 
même et quelles que soit les intentions du célébrant. L'efficacité de la prière ne dépend pas des dispositions intimes du fidèle, mais de l'exactitude des mots, du geste et de l'intonation. (...). Si une divinité est invoquée suivant les formes exactes, surtout si l'on sait prononcer son véritable nom, elle est contrainte d'agir selon la volonté de son prêtre. Les paroles sacrées sont une incantation qui soumet les puissances supérieures à l'officiant, quel que soit le but qu'il veuille atteindre ». Le livre XI des Métamorphoses d'Apulée, qui clôt le roman et qui culmine dans les initiations isiaques qui terminent l'ouvrage, en témoigne encore. Au chapitre seize, Lucius, sur le point d'être initié, s'approche d'un navire « dont la voile brillante (...) portait en évidence des lettres brodées en or qui étaient l'expression des vœux pour l'heureuse reprise de la navigation. » Ensuite (chapitre 17), Lucius et ses amis arrivent aux abords du temple de la déesse. Le Grammateus prononce alors des voux de prospérité, d'après un texte écrit. Enfin, au moment de préparer l'initiation, le prêtre conduit le protagoniste jusqu'à la porte du temple et apporte des livres précieusement conservés dans une cachette située au fond du temple. Ces livres, destinés à instruire le myste en vue de l'initiation, comportaient des caractères inconnus, figures d'animaux qui exprimaient des formules liturgiques, des traits noueux ou arrondis, bref, en d'autres termes, des hiéroglyphes, qui protégeaient le texte de la curiosité des profanes (a curiositate profanorum lectione munita) ${ }^{33}$. C'est ainsi aussi que s'explique le rôle des Ephesia grammata.

Cette idée de protectionnisme, propre au paganisme, sera combattue par la pensée chrétienne, qui défend l'universalité de la divinité, révélée à tous les hommes. Dans le Contre Celse ${ }^{34}$, Origène répond à une objection que lui avait

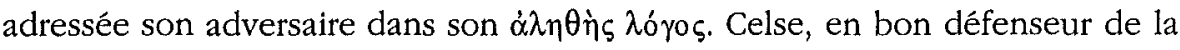
conception païenne, prétendait que les prières formulées en langue barbare étaient efficaces, tandis que celles récitées en grec ou en latin ne l'étaient pas. Origène rétorque que, si les Grecs prient en grec et les Latins en latin, chaque peuple prie dans la langue qui est la sienne, sans que la langue utilisée ait une influence sur l'efficacité de la prière. Voyons le texte :

33 Chap. 27, 8. - L'influence du rituel magique pratiqué dans les temples égyptiens de l'époque ptolémaïque et romaine se fait jour à plusieurs reprises dans le roman, ainsi qu'en témoigne, par exemple, l'affaire du marché d'Hypata (I, 24, 3-25, 6), étudiée par Ph. DERCHAIN et J. HuBAuX, L'affaire du marché d'Hypata dans les Métamorphoses d'Apulée, in AC, 27 (1958), p. 100-104.

34 vill, 37. 
Si on prononce leurs noms dans une langue barbare, ils auront de la puissance, mais si c'est en grec ou en latin, ils n'en auront pas [critique de Celse]. Qu'on nous montre donc celui dont nous invoquons le nom dans une langue barbare pour l'appeler à l'aide ! On verra bien l'insanité de cette critique de Celse en constatant que la foule des chrétiens n'use même pas dans ses prières des noms qui sont littéralement dans les divines Écritures pour désigner Dieu, mais que les Grecs se servent de mots grecs, les Romains de mots latins et ainsi chacun selon sa propre langue, pour prier Dieu et le louer comme il peut. Et le Seigneur de toute langue écoute ceux qui prient en chaque langue, comme s'il écoutait une voix pour ainsi dire unique en ce qu'elle veut signifier, bien qu'elle s'exprime en diverses langues. Car le dieu suprême n'est pas un de ceux qui ont en héritage une langue barbare ou grecque, ignorant les autres et n'ayant aucun souci de ceux qui parlent d'autres langues.

(Traduction M. Borret [Paris, 1969 = Sources chrétiennes, 150]).

Le conservatisme visant à perpétuer un privilège et un secret est une attitude propre aux classes sociales élevées, jalouses de leur passé. Elles entendent conserver à toute force une religion héritée de leurs ancêtres les plus prestigieux, dont les rois et les princes d'autrefois furent les dépositaires, et refusent de partager leur foi avec le peuple, a fortiori avec les étrangers. Cette attitude conduit vers la constitution d'une religion hermétique qui n'admet qu'un certain nombre d'initiés qui peuvent communier à ses mystères. Elle implique la plupart du temps une initiation qui exclut la majorité de la population et devient par ce biais la religion d'une élite. C'est là un schéma de pensée que l'on trouve aussi dans la Grèce classique, comme en témoigne le prologue de l'œuvre de Parménide d'Élée, où la connaissance de la vérité se trouve au bout d'un chemin, qui conduit l'initié au royaume de la lumière. Comme le dit W. Jaeger ${ }^{35}$, « la vision de cette mystérieuse entrée au royaume de la lumière est une authentique expérience religieuse. »

L'attitude inverse, en revanche, est le signe d'une volonté de partager avec le monde entier un «évangile». Elle implique une perméabilité aux cultures étrangères que permet l'ouverture au monde caractéristique de l'époque hellénistique. Les conquêtes d'Alexandre ont fait prendre conscience aux Grecs, qui vivaient jusque-là en autarcie culturelle, de l'existence d'autres cultures et d'autres systèmes de pensée que les leurs et du profit qu'ils pouvaient tirer de leur connaissance ${ }^{36}$. Cette perméabilité, qui n'implique pas nécessairement une traduction, n'est pas à sens unique. Si la Grèce reçoit en héritage le panthéon

\footnotetext{
35 À la naissance de la Théologie. Essai sur les Présocratiques, Paris, 1966, p. 105.

36 Voir les contributions réunies dans le volume intitulé Grecs et barbares (Fondation Hardt, vol. VIII, Genève-Vandœuvres, 1961).
} 
égyptien, comme en témoigne déjà la Théogonie d'Hésiode, qui assimile mécaniquement les dieux égyptiens aux divinités grecques correspondantes, la littérature égyptienne tardive témoigne d'une influence de la Grèce. Ainsi cette sorte d'épopée mettant en scène des dynastes locaux qu'est le Cycle de Petoubastis a subi, dès son apparition, vers 650 av. J.-C., l'influence de l'Iliade, probablement amenée en Égypte par les fondateurs de Naucratis ${ }^{37}$, et, plus tard, le Roman de Siosiri s'inspirera d'un récit pythagoricien de Descente aux Enfers, qui remonte vraisemblablement à Héraclide du Pont $\left(l^{\prime} \text { Abaris }\right)^{38}$, Du point de vue grec, la traduction dans la langue des Hellènes de plusieurs textes religieux de l'Égypte favorise le passage dans la pensée grecque de maints aspects de la théologie égyptienne. Cette volonté apostolique, grâce à laquelle la religiosité de l'Égypte "passe » en Grèce, concerne les manifestations les plus populaires des cultes. C'est cette voie qu'emprunte Isis, qui parviendra finalement en terre italique. C'est cette voie aussi que prennent les dieux d'Asie mineure, dont la Grande Mère Phrygienne, Cybèle, ou encore les cultes de Syrie $^{39}$. C'est ce même esprit apostolique, comparable à celui des missionnaires du christianisme naissant, qui animera encore, aux confins de l'hellénisme, la traduction en grec des édits du roi Maurya Açoka, l'apôtre du bouddhisme parmi les Grecs $^{40}$. Seule la perte de ces territoires lointains lors du partage du royaume d'Alexandre après sa mort explique que cette religion ne parvînt pas en Grèce. Enfin, c'est une volonté comparable qui explique le passage de la science des Chaldéens, l'astronomie et l'astrologie, qu'achemineront dans la pensée grecque les «mages hellénisés ${ }^{41}$.

37 J. SCHWARTZ, Les conquérants perses et la littérature égyptienne, in BIFAO, 48 (1948), p. 6580 et Le "Cycle de Petoubastls" et les commentaires égyptiens de l'Exode, in BIFAO, 49 (1949), p. 67-83 ; Ph. DERCHAIN, Miettes, in REgypt, 26 (1974), p. 7-20 (spéc. 15-19). Plus généralement, A. LOPRIENO, Topos und Mimesis. Zum Auslander in der dgyptiscben Literatur, Wiesbaden, 1988 (Ägyptologische Abb. 48).

38 I. LÉvY, La légende de Pythagore de Grèce en Palestine, Paris, 1927, p. 171-209 (spéc. p. 197 198).

39 F. CUMONT, Lux Perpetua, Paris, 1949, p. 259-274.

10 A.-J. FESTUGIÈRE, Les inscriptions d'Ašoka et l'idéal du rol bellénistique, in RecSR, 39/40 (1951-52) (= Mélanges Lebreton I), p. 31-46 ; E. BENVENISTE, Edits d'Asoka en traduction grecque, in JA, 252 (1964), p. 137-157 ; G. Pugliese Carratelli et alit (éds), Un editto bilingue greco-aramatco di Ašoka. La prima iscrizione greca scoperta in Afghanistan, Roma, 1958 (Serie Orientale Roma, 21) [version anglaise : A bilingual Graeco-Aramaic edict by Asoka. The First Greek Inscription discovered in Afghanistan [...], Roma, 1964 (Serie Orientale Roma, 29)] ; D. SCHLUMBERGER, Une nouvelle inscription grecque d'Açoka, in CRAI (1964), p. 126-34 [version remaniée dans $\mathrm{F}$. Altheim et alil (éds), Der Hellenismus in Mittelasien, Darmstadt, 1979 (WdF, 91), p. 406-417] ; G.R. FRANCI, Asoka at confini dell'ellenismo [...], in Mnemosynum. Siudi in onore di Alfredo Ghiselli, Bologna, 1989 , p. 225-33.

41 J. BIDEZ et F. CUMONT, Les mages belléntsés. Zoroastre, Ostanès et Hystaspe d'après la tradition grecque, 2 vol., Paris, 1938. 
Si la pensée hermétique plonge ses racines dans la conception grecque archaïque, qui connaît, pour l'avoir inventée, la notion de parole-acte, l'idée de transmettre un savoir, qu'il soit religieux ou non, - cela ne change rien au problème, - est une caractéristique de la sophistique du Ve siècle, qui, déjà consciente des limites de l'hellénisme et de l'existence d'autres domaines linguistiques ${ }^{42}$, trouvera un terrain favorable pour se développer dans la philosophie cosmopolite des systèmes hellénistiques. On la trouve aussi dans la dialectique socratique ${ }^{43}$ et, plus généralement, dans la pensée de Platon. Ainsi, dans le Cratyle $e^{44}$, lorsqu'il développe la thèse du «nom-convention », c'est, sans y faire allusion et, sans doute, sans en être conscient, la légitimité de la traduction que défend Hermodore.

Ces deux courants de pensée relatifs à la traduction illustrent l'existence de deux types de religiosités présentes dans l'Égypte gréco-romaine. L'une est réservée à la classe dirigeante et ne peut être communiquée aux non-initiés. Centrée sur l'individu, cette théologie est révélée à un petit groupe de privilégiés, qui refusent de la transmettre aux autres en traduisant les textes sur lesquels elle se fonde. Elle conduira à la religion absconse des Astrologues et aux mystères de la gnose. Comme l'écrit $\mathrm{F}$. Cumont ${ }^{45}$, « ces livres profonds [i.e. ceux des Astrologues], d'une intelligence difficile, ne pouvaient être communiqués aux profanes. Seule une élite, qui s'en était montrée digne, devait participer à cette science ésotérique, dont les rois et les prêtres d'autrefois avaient été les premiers dépositaires. " Cette religion occulte suscitera la méfiance des autorités qui prendront des mesures pour qu'elle reste inoffensive. Septime Sévère, rapporte Dion Cassius ${ }^{46}$, fit enfermer tous les livres secrets d'Égypte dans le tombeau d'Alexandre, à Alexandrie. D'autre part, le caractère intraduisible des textes religieux égyptiens tient aussi dans le surcodage linguistique entraîné par les hiéroglyphes eux-mêmes qui, comme le rappelle Thoth dans le traité XVI du Corpus Hermeticum, comportent une

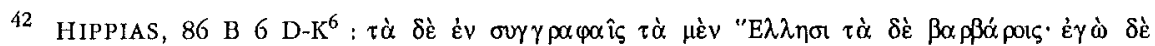

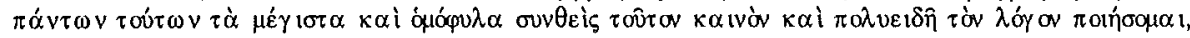
«dans les récits en prose, [on trouve ces thèmes] pour une part chez les Grecs et pour une autre chez les barbares. Dans tout ce matériau, j'ai choisi les récits les plus importants et les plus suivis pour en faire le traité que voici, nouveau et aux facettes multiples ».

43 PLATON, Gorgias, 471-472 et Ménon, 82a-86c.

$44385 \mathrm{c}$ : «je puis appeler chaque objet de tel nom établi par moi ; toi, de tel autre, établi par toi. Il en est de même pour les cités. Je les vois parfois assigner chacune un nom différent aux mêmes objets, des Grecs se séparer ainsi des autres Grecs et les Grecs des Barbares. » (Traduction L. Méridier [Paris, 1931]).

45 F. CUMONT, L'Égypte des Astrologues, Bruxelles, 1937, p. 152.

46 LXXV, 132. 
valeur performative, La gravure des hiéroglyphes, qui n'est pas une écriture comme une autre, est ressenti comme un acte cultuel permanent, car cette écriture signifie plus que les sons ${ }^{47}$.

L'autre pan de la pensée religieuse, qui concerne plutôt les cultes populaires, est destiné à la communauté universelle des hommes, quelles que soient leur nationalité et la langue qu'ils parlent. En voulant communiquer à tous les hommes un message de salut, elle préfigure l'esprit triomphant qui animera les premières communautés chrétiennes et les missionnaires chargés d'évangéliser le monde, même si leurs connaissances linguistiques ne sont pas toujours étendues ${ }^{48}$. Tout à fait éclairant à cet égard est le rapprochement entre l'idéal d'universalité du Dieu chrétien décrit par Origène (« car le Dieu suprême n'est pas un de ceux qui ont en héritage une langue barbare ou grecque, ignorant les autres et n'ayant aucun souci de ceux qui parlent d'autres langues ») et l'hymne d'Isis du temple de Medinet-Madi, qui exprime la même conception universelle de la divinité, au-delà des différences linguistiques ${ }^{49}$.

La fortune de la traduction dans les milieux religieux de l'Égypte grécoromaine connaîtra des prolongements dans le domaine copte, où se poseront de nouveaux problèmes. En effet, l'apparition du copte, - forme que prit la langue égyptienne, en adoptant un système d'écriture largement inspiré de l'alphabet grec, - et la naissance consécutive d'un trilinguisme gréco-latinocopte, particulièrement sensible dans les milieux monastiques ${ }^{50}$, donna lieu à la constitution de toute une littérature chrétienne copte, dont l'essentiel consista dans des traductions de Pères grecs, notamment de Jean Chrysostome ${ }^{51}$. Ces traductions du grec en copte, que l'on peut confronter aux originaux, comporte

47 Sur la spécificité de l'écriture hiéroglyphique, cf. Ph. DERCHAIN, Les biéroglyphes à l'époque ptolématque, in $\mathrm{Cl}$. Baurain - C. Bonnet et V. Krings (éds), Pbointkeia grammata. Lire et écrire en Méditérannée. Actes du Colloque de Liège (15-18 novembre 1989), Namur, 1991 (Collection d'Études classiques, 6), p. 243-356.

48 H. HENGEL, Juden, Griecben und Barbaren, Stuttgart, 1976 (Stuttgarter Bibelstudien, 76), p. 88. Plus généralement, Ch. MOHRMANN, Das Spracbenproblem in der fribcbristlicben Mission, in $Z M K, 38$ (1954), p. 103-111.

$49 S E G$, VIII, 548, 1. 14-17 (cf. V.F. VANDERLIP, The Four Greek Hymns of Istdorus and the Cult

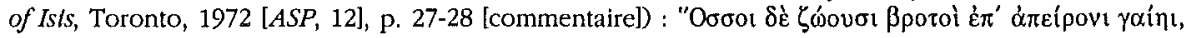

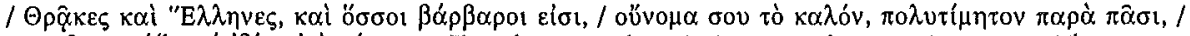

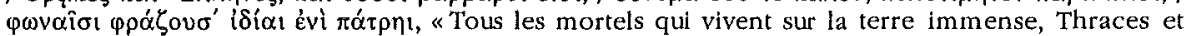
Grecs et tous les barbares, prononcent ton beau nom, très vénéré chez tous les hommes, chacun dans sa langue, chacun dans sa patrie».

50 G. BARDY, La question des langues dans l'Église ancienne, Paris, 1948, p. 38-52 et, plus particulièrement sur les connaissances linguistiques des moines d'Égypte, A.-J. FESTUGIÈRE, Les moines d'Orient, I (Paris, 1961), p. 23-25 et 77, n. 4.

51 Voir le recueil édité par P. NAGEL, Probleme der koptischen Literatur, Halle-Wittenberg, 1968 (Wiss. Beitrige der MLU Halle-Wittenberg). 
de nombreux termes grecs translittérés et témoignent des difficultés à exprimer en copte des notions théologiques spécifiques au grec. La connaissance du grec disparut d'ailleurs assez vite dans les milieux sacerdotaux, comme le montre le Testament d'Abrabam (vers 600), évêque d'Harmant en Haute-Égypte, rédigé en copte et finalement traduit en grec, pour des raisons d'ordre administratif ${ }^{2}$.

L'Égypte des Ptolémées était déjà familiarisée avec la traduction de textes à contenu religieux. Qu'il suffise de rappeler la version grecque du texte de propagande connu sous le nom de Oracle du Potier, réalisée vers 130 av. J.-C. ${ }^{53}$ Même si les techniques utilisées par les traducteurs nous échappent totalement, on perçoit les difficultés qu'ils éprouvent. En témoigne la formule $\kappa \alpha \tau \grave{\alpha}$ rò $\delta$ vo $\alpha$ cóv qui accompagne beaucoup de textes traduits.

Lieu de rencontre entre les cultures et les langues ${ }^{54}$, l'Égypte grécoromaine, héritant d'une longue tradition de bilinguisme ${ }^{55}$, prolongea et développa les contacts avec l'hellénisme, dont elle subit, en retour, l'influence. La problématique de la traduction de textes à contenu religieux illustre la manière dont l'Égypte assura la continuité de la tradition et le maintien de la parole sacrée, inaugurée en Grèce archaïque. Elle montre comment ce protectionnisme religieux fut à l'origine de la pensée hermétique de la fin de l'antiquité. Mais, en développant un courant opposé visant à partager un message avec

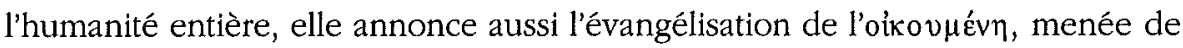
façon triomphante, telle qu'elle apparaît par exemple dans l'action de saint

52 P. Lond. I, 77.

53 G. MANTEUfFel, De opusculis Graecis, p. 99-106 et F. DUNAND, L'Oracle du Potier, in L'Apocalyptique, Paris, 1977, p. 36-67.

54 Sur les contacts interculturels en Égypte, cf. J. YOYOTTE, Bakbtis, Religion égyptienne et culture grecque à Edfou, in Religions en Égypte bellénistique et romaine. Colloque de Strasbourg (16-18 mai 1967), Paris, 1969 (Bibliotbèque des Centres d'Études supérieures spéclalisées Travaux du Centre d'Études supérieures spécialisées d'Histotre des Religions de Strasbourg), p. $127-141$.

55 W. PEREMANS, Uber die Zweisprachigheit im ptolemalschen Ägypten, in Festschrift Ortel, Bonn, 1964, p. 49-60; Le bilinguisme dans les relations gréco-égyptiennes sous les Lagides, in Egypt and Hellentstic World. Proceedings of the Int. Colloquium, Leuven, 1983 (Studia bellenist., 27), p. 253-280; Le bilinguisme dans l'Égypte des Lagides, in Studia Naster, II (Orientalla antlqua), Leuven, 1982 (Orient. Lovan. Analecta, 13), p. 143-154; M.C. BETRÒ, L'alcbimia delle traduztoni: il mito dell'Occhio del Sole e $l$ l PB. M. INV. No 274, in Atti del XVII congresso int. di Papirologia, III, Napoli, 1984, p. 1355-1360. 
Paul $^{56}$, et elle préfigure la traduction qui lui fera suite des Évangiles dans les idiomes du monde.

Bruno ROCHETTE

Université de Liège Aspirant du F.N.R.S.

Philologie classique

Place du 20-Août, 32

B - 4000 LIÈGE

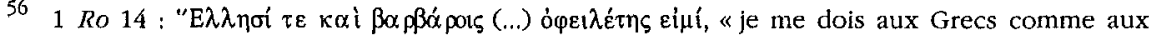
barbares ». Sur la notion antithétique "E $\lambda \lambda \eta v \varepsilon \varsigma / \beta \alpha \alpha \beta \beta \alpha \rho 0 t, c f .$, parmi une bibliographie abondante, I. OPELT et W. SPEYER, art. Barbar, in Nacbtrage zum RLAC, JbAC, 10 (1967), p. 251-290 (avec une importante bibliographie). 